\title{
Barriers to Adult Cochlear Implant Care in the United States: An Analysis of Health Care Delivery
}

\author{
Ashley M. Nassiri, M.D., M.B.A., ${ }^{1}$ John P. Marinelli, M.D. ${ }^{2}{ }^{2}$ \\ Donna L. Sorkin, M.A., ${ }^{3}$ and Matthew L. Carlson, M.D. ${ }^{1,4^{\prime}}$
}

ABSTRACT

Persistent underutilization of cochlear implants (CIs) in the United States is in part a reflection of a lack of hearing health knowledge and the complexities of care delivery in the treatment of sensorineural hearing loss. An evaluation of the patient experience through the CI health care delivery process systematically exposes barriers that must be overcome to undergo treatment for moderate-to-severe hearing loss. This review analyzes patient-facing obstacles including diagnosis of hearing loss, CI candidate identification and referral to surgeon, CI evaluation and candidacy criteria interpretation, and lastly CI surgery and rehabilitation. Pervasive throughout the process are several themes which demand attention in addressing inequities in hearing health disparities in the United States.

KEYWORDS: cochlear implant, hearing loss, barriers to care, patient experience, health disparities

\begin{abstract}
Although increasingly associated with significant health and socioeconomic ramifications, hearing loss remains one of the most undertreated disabilities in the United States. ${ }^{1,2}$ Within the hearing-impaired population, approximately 1.3 million individuals are estimated to meet cochlear implant (CI) candidacy under traditional bilateral severe-to-profound
\end{abstract}

sensorineural hearing loss (SNHL) criteria, though treatment with cochlear implantation among the candidate population is far from routine practice. Estimates of CI prevalence among the candidate population in the United States vary, ranging from 2.1 to $12.7 \%{ }^{3}$; however, even optimistic assessments utilizing more stringent candidacy criteria reflect a low
${ }^{1}$ Department of Otolaryngology - Head and Neck Surgery, Mayo Clinic, Rochester, Minnesota; ${ }^{2}$ Department of Otolaryngology - Head and Neck Surgery, San Antonio Uniformed Services Health Education Consortium, San Antonio, Texas; ${ }^{3}$ American Cochlear Implant Alliance, McLean, Virginia; ${ }^{4}$ Department of Neurologic Surgery, Mayo Clinic, Rochester, Minnesota.

Address for correspondence: Matthew L. Carlson, M.D., Department of Otolaryngology - Head and Neck
Surgery, Mayo Clinic, 200 1st St SW, Rochester, MN 55905 (e-mail: carlson.matthew@mayo.edu).

Barriers to Cochlear Implant Access: Acknowledging the Challenges, Changing the Future; Guest Editor, Sarah Sydlowski, Au.D., Ph.D., M.B.A.

Semin Hear 2021;42:311-320. (C) 2021. Thieme. All rights reserved. Thieme Medical Publishers, Inc., 333 Seventh Avenue, 18th Floor, New York, NY 10001, USA DOI: https://doi.org/10.1055/s-0041-1739281.

ISSN 0734-0451. 
prevalence. ${ }^{2,4-14}$ Despite growing recognition of the underutilization of CIs in the last decade, recent estimates suggest nearly flat prevalence curves without substantial growth of the percentage of implanted individuals within the CI candidate cohort. $^{3}$

Poor CI utilization in part reflects the lack of widespread hearing health knowledge and the complexities of care delivery in the treatment of SNHL. From a patient perspective, the process that spans diagnosis of hearing loss to rehabilitation involves a spectrum of individual, locoregional, and systemic barriers which can discourage appropriate referral and treatment. ${ }^{15,16}$ An evaluation of the patient experience via a detailed analysis of the hearing health care delivery model can systematically expose the barriers associated with each aspect of the CI care delivery process (Fig. 1). While the barriers that patients encounter vary on an individual basis, this review broadly describes major barriers to adult CI care in the United States from the patient perspective.

\section{THE COCHLEAR IMPLANT PROCESS}

\section{Diagnosis and Screening of Hearing Loss}

The initial obstacle patients must overcome in the cochlear implantation pathway is receiving an accurate diagnosis that identifies them as a potential candidate. Several studies have demonstrated that patients oftentimes wait for years with qualifying hearing loss prior to undergoing cochlear implantation. ${ }^{9,17,18}$ In a multicenter trial across 14 institutions in the United States, patients' average duration of severe-to-profound SNHL exceeded 10 years. ${ }^{17}$ Recent studies characterizing the audiometric profiles of CI recipients at large CI centers corroborate this claim, showing most modern $\mathrm{CI}$ recipients experience years of qualifying hearing loss resulting in average scores between 10 and 15\% correct on consonant-nucleus-consonant (CNC) word and AzBio sentence testing with pure-tone averages (PTAs) around $90 \mathrm{~dB}$ on initial CI evaluation. ${ }^{9,18}$ Unfortunately, patients' delay in diagnosis leads to excess years lived with unnecessarily severe hearing disability while also negatively influencing device performance outcomes, as duration of deafness and preoperative speech recognition scores represent some of the few consistent predictors of postoperative audiometric outcomes. ${ }^{19,20}$

Several patient factors influence the observed delay in diagnosis. Behind the general lack of awareness surrounding cochlear implantation, there exists an overarching, poor appreciation for hearing loss as a disease that requires treatment among the general patient populace. ${ }^{21-23}$ Often accepted as an inevitable sequela of senescence, the lacking literacy

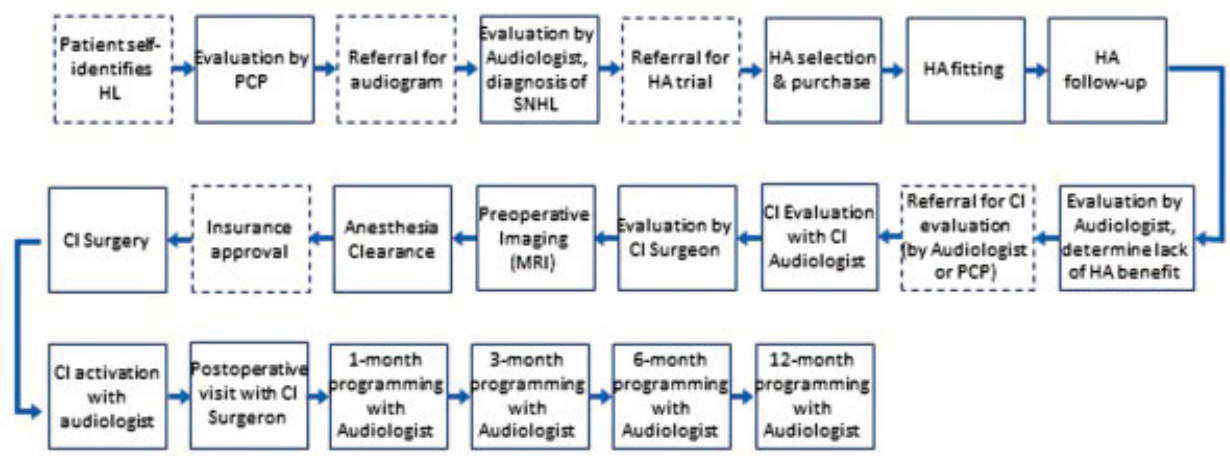

Figure 1 Cochlear implant process map. This process map outlines one simplified variation of the steps necessary to undergo cochlear implantation for a candidate patient who self-identifies hearing loss. Solid boxes represent an appointment, and dashed boxes represent events that need to occur prior to the next appointment. In this typical example, the patient must attend 15 appointments from initial diagnosis of hearing loss to completion of 1 year of postoperative programming. $\mathrm{HL}$, hearing loss; $\mathrm{PCP}$, primary care physician; $\mathrm{SNHL}$, sensorineural hearing loss; $\mathrm{HA}$, hearing aid; $\mathrm{Cl}$, cochlear implant; $\mathrm{MRI}$, magnetic resonance imaging. 
regarding negative long-term health outcomes provides little impetus for most patients to actively seek treatment. ${ }^{24}$ In a 2014 National Health Interview Survey of 40.3 million adults with self-reported hearing loss, 12.9 million (32\%) had never seen a health care professional regarding their hearing loss, and more than 11 million (28\%) had never previously undergone formal audiometric testing. ${ }^{23}$ Moreover, patients' self-reported hearing loss often underestimates the true severity of their disability, ${ }^{25}$ thus rendering the likelihood of patients drawing attention to their hearing loss during a short visit with their primary care provider even more remote. Sociodemographic factors, such as patient's age and race, also appear to influence the likelihood of referral for CI evaluation and implantation. ${ }^{8,18,26,27}$ For instance, elderly patients have been shown to be less likely to receive a CI despite meeting audiometric criteria, ${ }^{8,18,26}$ and non-white patients could be as much as six times less likely to undergo cochlear implantation per year of hearing loss compared with patients self-identifying as white. ${ }^{8,26}$ Taken together, multiple patient-related factors influence their likelihood of voluntarily drawing attention to an untreated or inadequately treated hearing disability within routine health care encounters with primary care providers.

Frontline providers' limited familiarity with hearing norms and indications for cochlear implantation further compounds the challenge of identifying disabling hearing loss within a short office visit. Unlike the widely adopted and equally successful screening for newborn hearing loss, there exists no institutionalized screening program for adults at most centers in the United States. ${ }^{28}$ Unfortunately, it is estimated that only $15 \%$ of frontline providers actively screen for hearing loss during routine clinical encounters. ${ }^{29}$ As a consequence, only $3 \%$ of CI recipients report receiving their initial referral by their primary care provider. ${ }^{30,31}$ Despite the aforementioned limitations of self-reported hearing loss among patients, the most recent United States Preventative Services Task Force statement could not recommend routine screening in adults not complaining of subjective hearing loss due to insufficient evidence of benefit. ${ }^{25,28}$ In the absence of institutionalized screening programs, patients oftentimes do not receive referrals to audiology practices for testing. ${ }^{32}$ Moreover, audiology practices are seldom linked to primary care offices, and although available, there exists limited use of remote, app-based assessment of patients' hearing to date. ${ }^{33}$ Practically, the requirement for referral introduces travel and health care reimbursement limitations to patients with disabling hearing loss being correctly identified. Lastly, in the event the patient is not referred to a CI center by the audiologist, it is improbable that the frontline provider possesses adequate training to allow for interpretation and resultant referral based on the obtained audiometric data. $^{34}$

\section{Potential Candidate Identification and Referral to Surgeon}

Reflective of misconceptions and variable practice patterns even amidst general otolaryngologists, among those with a diagnosis of severe-to-profound SNHL, few patients are referred to CI surgeons for CI evaluation. ${ }^{4,35}$ Recognition of potential CI candidacy and appropriate referral to a CI surgeon comprise the next, and arguably most substantial, set of barriers for patients with significant hearing impairment. While the factors influencing candidate identification and referrals are numerous, misconceptions of evolving CI candidacy criteria drive medical decision-making even among audiologists and general otolaryngologists. Despite major technological and surgical advances that have led to the expansion of CI criteria to additionally include those with moderate and asymmetric SNHL, the implementation of expanded criteria in the clinical setting remains highly variable across $\mathrm{CI}$ otolaryngology and audiology practices. ${ }^{7,9,36-38}$ Surveys of general practice audiologists underscore this point, as most audiologists report seeing and referring fewer CI candidates in their practice than would be expected based on the prevalence of severeto-profound SNHL among patients evaluated in an audiology clinic. ${ }^{39}$ Remarkably, only 3\% of all patients with moderate-to-profound SNHL are referred for CI evaluation. ${ }^{4}$ Despite expanded CI criteria, Holder et al demonstrated that even at a progressive, high-volume academic center, patients who underwent CI 
had an average CNC score of $10 \%$ and PTA of $89 \mathrm{~dB}$, suggesting significant delayed treatment effect. ${ }^{9}$ In total, although CI criteria have expanded, referrals for CI evaluation in patients with severe-to-profound SNHL are not routine practice, let alone in patients with asymmetric or moderate SNHL.

Indicative of the complexities of evolving CI candidacy criteria, there exist no definitive audiogram-based referral criteria for potential candidates to undergo CI evaluation. ${ }^{40} \mathrm{Few}$ studies have evaluated the value and accuracy of routine audiometry using PTA thresholds and monosyllabic word recognition testing in predicting CI candidacy. ${ }^{41-45}$ Gubbels et al presented a range of PTA values and word recognition scores and associated positive predictive values for meeting CI candidacy based on varying insurance criteria. ${ }^{41}$ While this study demonstrated a predictive association between routine audiometric measures and CI candidacy as $86 \%$ of patients with word recognition scores less than $33 \%$ met CI candidacy criteria on further evaluation, it did not recommend specific thresholds for audiometric CI candidacy screening. In an effort to present simplified audiometric criteria optimized for a CI candidacy screening process, Zwolan et al presented the "60/60 Guideline" for referring adults with Medicare insurance for CI evaluation, which reached sensitivity and specificity rates of 96 and $66 \%$, respectively. ${ }^{42}$ Unique to the CI field and critical to the development of effective referral guidelines, insurers have highly variable coverage criteria, ranging from typically less stringent private payers to more restrictive Medicare and Medicaid. ${ }^{46}$ As Sydlowski and Weaver emphasized, screening guidelines must take insurance coverage into consideration, as more stringent guidelines such as the " $60 / 60$ Guideline” developed for Medicare patients may deny individuals a referral who qualify under private insurer criteria. ${ }^{45}$ Ultimately, consensus guidelines for CI referral require consideration of audiometric measures and candidacy criteria based on insurance coverage. An effective screening instrument will remove the decision-making burden from primary care providers and general audiologists by providing clear, concise guidelines for referral for CI evaluation.
In addition to consistently identifying potential CI candidates, patient's progress through the CI care delivery model is dependent on frontline providers and general audiologists to discuss CI options with the patient and place appropriate referrals to CI surgeons and audiologists for further evaluation. This step, specifically encouraging referral and placing a referral request, can be a significant barrier to care for many patients. Even in cases where potential candidates are identified at a high rate, referral rates may be low, connecting a yield loss of nearly $40 \%$ at the time of CI discussion with the diagnosing audiologist. ${ }^{35}$ Several barriers at this time point have been implicated including misconceptions about surgery and potential benefits, other medical priorities, and satisfaction with current hearing aids. Among general audiologists, there exists considerable variability in referral patterns related to training, familiarity with the process, involvement in CI care on a professional level, and relationships with CI surgeons. ${ }^{47}$ In audiology practices without preestablished referral pathways for CI evaluation or in those without local CI support, appropriate referrals may be delayed or altogether obstructed. ${ }^{39}$ This barrier may be more significant in rural areas where CI centers and surgeons are relatively scarce. ${ }^{47-49}$ Along with clear referral guidelines, the establishment of defined referral options, especially in geographical regions with limited access to CI care, may reduce barriers to CI care for patients.

\section{Cochlear Implant Evaluation and Candidacy}

Variable practice patterns both in the methodology of CI evaluation testing and interpretation of CI candidacy criteria culminate in existing disparities and barriers to care across the United States. Tolisano et al demonstrated the real implications of testing variability in the "best-aided condition" using personal hearing aids compared with hearing aids fitted at the time of evaluation; patients fitted with a hearing aid at the time of evaluation were less likely to qualify for cochlear implantation and more likely to be non-white. ${ }^{27}$ Variability in practice among CI professionals extends to the interpretation and implementation of CI 
candidacy criteria put forth by insurers who leave criteria and testing requirements somewhat vague. This is particularly true in the case of speech perception testing in noise. Despite the substantial influence on CI candidacy qualification, speech perception testing in noise is not standardized across the United States, and practice variability includes extremes of the range from no testing in noise to $+5 \mathrm{~dB}$ signal-to-noise ratio utilized to qualify patients for CIs. ${ }^{37,50}$ It follows that patients with residual hearing who undergo CI evaluation without noise are less likely to qualify for CI compared with those who complete testing in noise. ${ }^{51}$ To compound the obstacles associated with CI candidacy testing, patient interviews have suggested that the testing battery does not represent real-world hearing difficulties and may have an impact on whether individuals decide to proceed with surgery. ${ }^{52}$

Even among CI audiologists and surgeons, there remains considerable controversy as to the interpretation and implementation of CI candidacy criteria. While a consensus statement has been developed for the management of traditional CI candidates, or those with bilateral moderate-to-profound SNHL, ${ }^{53}$ the management protocols for patients with significant residual hearing, asymmetric hearing loss, and single-side deafness are still debated. A survey of the American Neurotology Society highlighted the inconsistency in practice across CI surgeons with regard to patients who qualified for CI under expanded criteria. ${ }^{54}$ Not surprisingly, high-volume CI centers were more likely to perform off-label applications of CI, for example, in patients with single-side deafness or in children younger than 12 months. As CI candidacy criteria continue to evolve with technology and surgical techniques, barriers to care for some patient populations, particularly those traditionally associated with health disparities, are at risk of accentuation.

As alluded to previously, insurance coverage and CI candidacy criteria set forth by payers have considerable impact on access to care. Although uncommon, for most patients without insurance or with an insurer that does not cover cochlear implantation, undergoing evaluation and treatment is prohibitively expensive. When considering the ramifications of insurance coverage on access to CI care, the discrepancies between private insurers' criteria and more stringent Medicare criteria illustrate the unique barriers faced by Medicare beneficiaries. Contrary to most other insurance providers and FDA labeling, current Medicare stipulations require that patients demonstrate bilateral moderate-to-profound SNHL with sentence recognition scores no greater than $40 \%$ in the best-aided condition. ${ }^{55}$ These stringent requirements have meaningful implications, and can preclude $\mathrm{CI}$ in patients with considerable hearing impairment experiencing limited benefit from hearing amplification who do not meet the above criteria. $^{56}$ In cases of asymmetric hearing loss with a better hearing ear that does not meet Medicare stipulations, patients experience a delay in treatment for the qualifying ear as they wait for deterioration of the better hearing ear to ultimately meet bilateral, bestaided Medicare criteria, leading to unnecessary prolonged duration of deafness in the poorer hearing ear. ${ }^{18,57}$ As most adults aged more than 64 in the United States constitute the Medicare beneficiary population, unique bilateral criteria which restrict access to care and cause treatment delay do so in the population most vulnerable to significant hearing loss. ${ }^{58-60}$ Despite the incontrovertible benefits of CI in the elderly population, current Medicare criteria persistently discriminate against the geriatric population with hearing impairment. ${ }^{61-63}$

\section{Undergoing Cochlear Implantation and Rehabilitation}

The combination of inconsistent practices, poor general dissemination of information, and evolving technology has led to misconceptions about CIs among the patient population. While mainstream media has more recently featured CIs in films and commercials, ${ }^{64-66}$ a pervasive lack of general knowledge and accurate information impacts patients making treatment decisions. Despite increased use of both hearing aids and $\mathrm{CIs}$ in the last several decades, perceived stigma of hearing device use has a pervasive impact on patient decision-making throughout the hearing loss evaluation and 
treatment process. ${ }^{10,67}$ For patients interested in pursuing cochlear implantation, misinformation about costs and insurance coverage poses a barrier to pursuing treatment. In most cases, CIs, which are most often covered by insurance, involve less direct cost to the patient compared with hearing aids, which are traditionally an out-of-pocket cost for patients, although many patients assume the contrary. ${ }^{10,68}$ Unfortunately, CI cost coverage varies widely across payers and policies, and determination of the cost to the patient is frequently delayed until prior written authorization is approved or even postoperatively in many cases of private insurers. ${ }^{46}$ Additionally, surgical intervention for hearing treatment, as opposed to noninvasive hearing amplification, can present a desirability barrier for many patients. While in actuality a relatively routine procedure with rare complications, $\mathrm{co}^{-}$ chlear implantation is perceived as "brain surgery" among some patients, which carries a risky connotation and may delay or deter treatment. ${ }^{69}$ Anecdotally, the authors have encountered several patients who cite loss of residual hearing or concerns about sound quality as a deterrent, at least in part, for proceeding with surgery. While sound quality may be different compared with the acoustic side, speech perception outcomes will predominantly improve. Similarly, the authors have encountered patients who have been impacted by misinformation suggesting a negative impact of a CIhearing ear on binaural hearing, when, in fact, bimodal hearing typically outscores individual ears. Provision with upfront, accurate information early in the hearing loss evaluation and treatment process may be effective in overcoming informational barriers.

While most CI patients perceive significant benefit from their implants, variability in both patient expectations and the manner in which hearing health professionals set expectations and present outcomes data can have a considerable impact on patient decisionmaking. Objective CI outcomes data can be presented with different emphases which may influence patient decision-making. For example, a patient who is informed that nearly all patients achieve most speech performance growth by 1 year postoperatively may not be as enthusiastic as one who is informed that most patients perceive various hearing benefits by 6 months, or even sooner. While both statements are true, subjective framing can have a considerable impact on the interpretation of the data. Research specifically evaluating the role of the hearing health professional's attitude in both patient expectations and likelihood to proceed with $\mathrm{CI}$ is limited due to the difficulty in measuring subjective processes, although it is widely accepted that patient decision-making is dependent on the manner in which data are presented to them. ${ }^{70}$ Consequently, a surgeon or audiologist who presents more pessimistic outcomes data may inadvertently deter patients from proceeding with $\mathrm{CI}$.

For patients who decide to proceed with cochlear implantation, complexities of both the surgical and rehabilitative care delivery process may present overwhelming obstacles. For adult patients, presurgical workup (surgical evaluation, CI audiometric evaluation, anesthesia clearance, and imaging), CI surgery, and postoperative aural rehabilitation and programming require multiple appointments, up to 10, when uncoordinated. ${ }^{15,68}$ Aside from issues with convenience, the burden associated with these appointments can be an insurmountable barrier for patients requiring childcare, time off from work, and transportation assistance. ${ }^{15}$ Importantly, prior work has demonstrated considerable differences in utilization and access across patient geographic distribution, socioeconomic status, and age groups. ${ }^{4,18,48,49}$ As most insurance plans cover $\mathrm{CIs}$ for patients meeting criteria, geographic and informational barriers continue to limit access to care. ${ }^{71,72}$ While remote CI programming and telemedicine visits may offer some relief, these services are not routinely available for patients across the United States and state licensing laws often limit audiologic practice across state lines; consequently geographic distribution of CI centers limits accessibility. ${ }^{10,48,73-76}$ The relative scarcity of CI centers in part reflects the financial difficulties faced by practices offering $\mathrm{CI}$ care. A survey of $\mathrm{CI}$ surgeons and audiologists revealed that Medicare and Medicaid reimbursement rates failed to cover the costs associated with surgery and aural rehabilitation, thus financially disincentivizing practices to provide CI care. ${ }^{77}$ 
The COVID-19 pandemic has more recently encouraged an uptick in telemedicine; however, at the time of this publication, CI programming codes are only temporarily included for coverage under Medicare. For long-term success, a significant expansion of this type of coordinated care which leverages technology as well as regulatory updates would be required to bridge gaps in geographic distribution of CI centers. ${ }^{72,78,79}$

\section{CONCLUSIONS}

In an effort to improve CI utilization and direct resources effectively, a thorough understanding of patient-facing barriers to care is imperative. Mapping the patient experience is one way to uncover obstacles that CI candidates and recipients face at nearly every step of the process. Several themes permeate the CI care delivery process, which highlight targets for improvement. In the absence of a standardized audiologic screening process for adults, an initial diagnosis of hearing loss is a substantial barrier in seeking treatment. While hearing screening in the adult population is not routine practice in the United States currently, screening those atrisk for hearing loss may eliminate this burden altogether. Furthermore, lack of information or misinformation about hearing loss treatment options, CI referral criteria, and CI candidacy requirements are pervasive and impact patients, primary care providers, general audiologists, and general otolaryngologists. Development and dissemination of unambiguous, standardized CI referral guidelines removes the liability of identifying potential CI candidates from the primary care provider and general audiologist. Finally, for patients interested in pursuing hearing loss treatment or cochlear implantation, geographic and socioeconomic barriers to care limit access to CI centers. In some cases, coordinated care coupled with telemedicine utilizing remote programming and testing technology may alleviate the burden associated with the lengthy hearing loss evaluation and treatment process. Future efforts addressing these major barriers to CI care will need to take systemic and socioeconomic factors into account, in an effort to expand CI care equitably.

\section{FUNDING}

No funding or other support was required for this study.

\section{CONFLICTS OF INTEREST}

A.M.N.: Research funding from Cochlear Americas.

M.L.C.: Research funding from Cochlear Americas.

\section{REFERENCES}

1. Cunningham LL, Tucci DL. Hearing loss in adults. N Engl J Med 2017;377(25):2465-2473

2. NIDCD. National Institute on Deafness and Other Communication Disorders. Quick Statistics about Hearing. Accessed November 1, 2020 at: https:// www.nidcd.nih.gov/health/statistics/quick-statisticshearing\#10

3. Nassiri AM, Sorkin DL, Carlson ML. Current estimates of cochlear implant utilization in the United States. Otol Neurotol 2021; In press

4. Sorkin DL. Cochlear implantation in the world's largest medical device market: utilization and awareness of cochlear implants in the United States. Cochlear Implants Int 2013;14 Suppl 1 (Suppl 1)S4-S12

5. Sorkin DL, Buchman CA. Cochlear implant access in six developed countries. Otol Neurotol 2016;37 (02):e161-e164

6. Rapport F, Hughes SE, Boisvert I et al. Adults' cochlear implant journeys through care: a qualitative study. BMC Health Serv Res 2020;20(01):457

7. Varadarajan VV, Sydlowski SA, Li MM, Anne S, Adunka OF. Evolving criteria for adult and pediatric cochlear implantation. Ear Nose Throat J 2021;100(01):31-37

8. Tolisano AM, Schauwecker N, Baumgart B et al. Identifying disadvantaged groups for cochlear implantation: demographics from a large cochlear implant program. Ann Otol Rhinol Laryngol 2020;129(04):347-354

9. Holder JT, Reynolds SM, Sunderhaus LW, Gifford RH. Current profile of adults presenting for preoperative cochlear implant evaluation. Trends Hear 2018;22:2331216518755288

10. Barnett M, Hixon B, Okwiri N et al. Factors involved in access and utilization of adult hearing healthcare: a systematic review. Laryngoscope 2017;127(05):1187-1194

11. Cochlear Implant Candidacy for Adults. American Cochlear Implant Alliance. Published 2020. Accessed November 30, 2020 at: https://www. acialliance.org/page/AdultCandidacy 
12. US Market for Hearing Aids and Audiology Devices. iData Research Inc2010

13. US Market Report Suite for Hearing Devices. iData Research Inc2016

14. Gifford RH, Dorman MF, Shallop JK, Sydlowski SA. Evidence for the expansion of adult cochlear implant candidacy. Ear Hear 2010;31(02):186-194

15. Nassiri AM, Yawn RJ, Gifford RH et al. Same-day patient consultation and cochlear implantation: innovations in patient-centered health care delivery. Otol Neurotol 2020;41(02):e223-e226

16. Marinelli JP, Carlson ML. Barriers to access and health care disparities associated with cochlear implantation among adults in the United States. Mayo Clin Proc 2021;96(03):547-549

17. Balkany T, Hodges A, Menapace $\mathrm{C}$ et al. Nucleus Freedom North American clinical trial. Otolaryngol Head Neck Surg 2007;136(05):757-762

18. Barnes JH, Yin LX, Marinelli JP, Carlson ML. Audiometric profile of cochlear implant recipients demonstrates need for revising insurance coverage. Laryngoscope 2021;131(06):E2007

19. Carlson ML. Cochlear implantation in adults. $\mathrm{N}$ Engl J Med 2020;382(16):1531-1542

20. Semenov YR, Martinez-Monedero R, Niparko JK. Cochlear implants: clinical and societal outcomes. Otolaryngol Clin North Am 2012;45(05):959-981

21. D'Haese PSC, De Bodt M, Van Rompaey V, Van de Heyning P. Awareness of hearing loss in older adults: results of a survey conducted in 500 subjects across 5 European countries as a basis for an online awareness campaign. Inquiry 2018;55; 46958018759421

22. Disease GBD, Injury I, Prevalence CGBD 2016 Disease and Injury Incidence and Prevalence Collaborators. Global, regional, and national incidence, prevalence, and years lived with disability for 328 diseases and injuries for 195 countries, 1990-2016: a systematic analysis for the Global Burden of Disease Study 2016. Lancet 2017;390 (10100):1211-1259

23. Mahboubi H, Lin HW, Bhattacharyya N. Prevalence, characteristics, and treatment patterns of hearing difficulty in the United States. JAMA Otolaryngol Head Neck Surg 2018;144(01):65-70

24. Livingston G, Sommerlad A, Orgeta V et al. Dementia prevention, intervention, and care. Lancet 2017;390(10113):2673-2734

25. Angara P, Tsang DC, Hoffer ME, Snapp HA. Self-perceived hearing status creates an unrealized barrier to hearing healthcare utilization. Laryngoscope 2021;131(01):E289-E295

26. Dornhoffer JR, Holcomb MA, Meyer TA, Dubno JR, McRackan TR. Factors influencing time to cochlear implantation. Otol Neurotol 2020;41(02): 173-177

27. Tolisano AM, Fang LB, Kutz JW Jr, Isaacson B, Hunter JB. Better defining best-aided condition: the role of hearing aids on cochlear implantation qualification rates. Am J Otolaryngol 2020;41(03): 102431

28. Krist AH, Davidson KW, Mangione CM et al; US Preventive Services Task Force. Screening for hearing loss in older adults: US Preventive Services Task Force Recommendation Statement. JAMA 2021;325(12):1196-1201

29. Sorkin DL. Cochlear implantation in the world's largest medical device market: utilization and awareness of cochlear implants in the United States. Cochlear Implants Int 2013;14(Suppl 1): S4-S12

30. Bogardus ST Jr, Yueh B, Shekelle PG. Screening and management of adult hearing loss in primary care: clinical applications. JAMA 2003;289(15): 1986-1990

31. Sullivan F. Barriers in the ENT referral channel to the adoption of $\mathrm{CI}$ as a standard of care for severe to profound hearing loss patients. In: US Medical Devices Market Outlook. Published 2008. Accessed October 20, 2021 at: www.frost.com

32. Yueh B, Shapiro N, MacLean CH, Shekelle PG. Screening and management of adult hearing loss in primary care: scientific review. JAMA 2003;289 (15):1976-1985

33. Bright T, Pallawela D. Validated smartphonebased apps for ear and hearing assessments: a review. JMIR Rehabil Assist Technol 2016;3(02): e13

34. Klyn NAM, Letendre C, Shrestha N, Lambert BL, Dhar S. Interpretability of the audiogram by audiologists and physician non-specialists. Int J Audiol 2021;60(02):133-139

35. Looi V, Bluett C, Boisvert I. Referral rates of postlingually deafened adult hearing aid users for a cochlear implant candidacy assessment. Int J Audiol 2017;56(12):919-925

36. Arnoldner C, Lin VY. Expanded selection criteria in adult cochlear implantation. Cochlear Implants Int 2013;14(Suppl 4):S10-S13

37. Carlson ML, Sladen DP, Gurgel RK, Tombers NM, Lohse CM, Driscoll CL. Survey of the American Neurotology Society on Cochlear Implantation: Part 1, Candidacy assessment and expanding indications. Otol Neurotol 2018;39 (01):e12-e19

38. Huinck WJ, Mylanus EAM, Snik AFM. Expanding unilateral cochlear implantation criteria for adults with bilateral acquired severe sensorineural hearing loss. Eur Arch Otorhinolaryngol 2019;276 (05):1313-1320

39. Huart S. Unidentified and underserved: cochlear implant candidates in the hearing aid dispensing practice. Audiology Online2009

40. Hunter JB, Tolisano AM. When to refer a hearingimpaired patient for a cochlear implant evaluation. Otol Neurotol 2021;42(05):e530-e535 
41. Gubbels SP, Gartrell BC, Ploch JL, Hanson KD. Can routine office-based audiometry predict cochlear implant evaluation results? Laryngoscope 2017;127(01):216-222

42. Zwolan TA, Schvartz-Leyzac KC, Pleasant T. Development of a 60/60 guideline for referring adults for a traditional cochlear implant candidacy evaluation. Otol Neurotol 2020;41(07): 895-900

43. McRackan TR, Fabie JE, Burton JA, Munawar S, Holcomb MA, Dubno JR. Earphone and aided word recognition differences in cochlear implant candidates. Otol Neurotol 2018;39(07): e543-e549

44. Leigh JR, Moran M, Hollow R, Dowell RC. Evidence-based guidelines for recommending cochlear implantation for postlingually deafened adults. Int J Audiol 2016;55; (Suppl 2):S3-S8

45. Sydlowski SA, Weaver RE. Relationship between unaided word recognition scores and cochlear implant candidacy. American Cochlear Implant Alliance2021; Virtual Poster Presentation

46. Moses LE, Friedmann DR. Cochlear implant indications: a review of third-party payers' policies for standard and expanded indications. Cochlear Implants Int 2021;22(04):237-244

47. Hogan A, Taylor A, Westcott S. Audiologists' attitudes to cochlear implants. Cochlear Implants Int 2001;2(01):17-29

48. Hixon B, Chan S, Adkins M, Shinn JB, Bush ML. Timing and impact of hearing healthcare in adult cochlear implant recipients: a rural-urban comparison. Otol Neurotol 2016;37(09):1320-1324

49. Noblitt B, Alfonso KP, Adkins M, Bush ML. Barriers to rehabilitation care in pediatric cochlear implant recipients. Otol Neurotol 2018;39(05): e307-e313

50. Prentiss S, Snapp H, Zwolan T. Audiology practices in the preoperative evaluation and management of adult cochlear implant candidates. JAMA Otolaryngol Head Neck Surg 2020;146(02):136-142

51. Dunn C, Miller SE, Schafer EC, Silva C, Gifford $\mathrm{RH}$, Grisel JJ. Benefits of a hearing registry: cochlear implant candidacy in quiet versus noise in 1,611 patients. Am J Audiol 2020;29(04): 851-861

52. Athalye S, Mulla I, Archbold S. The experiences of adults assessed for cochlear implantation who did not proceed. Cochlear Implants Int 2014;15(06): 301-311

53. Buchman CA, Gifford RH, Haynes DS et al. Unilateral cochlear implants for severe, profound, or moderate sloping to profound bilateral sensorineural hearing loss: a systematic review and consensus statements. JAMA Otolaryngol Head Neck Surg 2020;146(10):942-953
54. Carlson ML, O'Connell BP, Lohse CM, Driscoll CL, Sweeney AD. Survey of the American Neurotology Society on Cochlear Implantation: Part 2, Surgical and device-related practice patterns. Otol Neurotol 2018;39(01):e20-e27

55. Decision Memo for cochlear implantation (CAG00107N). Centers for Medicare \& Medicaid Services2005. Accessed May 1, 2021

56. Zwolan TA, Kallogjeri D, Firszt JB, Buchman CA. Assessment of cochlear implants for adult Medicare beneficiaries aged 65 years or older who meet expanded indications of open-set sentence recognition: a multicenter nonrandomized clinical trial. JAMA Otolaryngol Head Neck Surg 2020;146(10):933-941

57. Miller SE, Anderson C, Manning J, Schafer E. Insurance payer status predicts postoperative speech outcomes in adult cochlear implant recipients. J Am Acad Audiol 2020;31(09):666-673

58. Lin FR, Niparko JK, Ferrucci L. Hearing loss prevalence in the United States. Arch Intern Med 2011;171(20):1851-1852

59. Lin FR, Thorpe R, Gordon-Salant S, Ferrucci L. Hearing loss prevalence and risk factors among older adults in the United States. J Gerontol A Biol Sci Med Sci 2011;66(05):582-590

60. Choi JS, Briggs SE. Health disparities in geriatric cochlear implantation. Am Acad Otolaryngol Head Neck Surg Bull2020:39

61. Issing C, Baumann U, Pantel J, Stöver T. Cochlear implant therapy improves the quality of life in older patients - a prospective evaluation study. Otol Neurotol 2020;41(09):1214-1221

62. Issing C, Baumann U, Pantel J, Stöver T. Impact of hearing rehabilitation using cochlear implants on cognitive function in older patients. Otol Neurotol 2021;42(08):1136-1141

63. Lally JW, Adams JK, Wilkerson BJ. The use of cochlear implantation in the elderly. Curr Opin Otolaryngol Head Neck Surg 2019;27(05):387-391

64. Marder D. Sound of Metal [Film]. Amazon Studios 2020

65. Cooley J. Toy Story 4 [Film]. Walt Disney Studios 2019

66. First Call With My Son - Matthew's True Pixel Story. YouTube.com2021

67. Wallhagen MI. The stigma of hearing loss. Gerontologist 2010;50(01):66-75

68. Laplante-Lévesque A, Hickson L, Worrall L. Factors influencing rehabilitation decisions of adults with acquired hearing impairment. Int J Audiol 2010;49(07):497-507

69. Bierbaum M, McMahon CM, Hughes S et al. Barriers and facilitators to cochlear implant uptake in Australia and the United Kingdom. Ear Hear 2020;41(02):374-385

70. Kassirer S, Levine EE, Gaertig C. Decisional autonomy undermines advisees' judgments of 
experts in medicine and in life. Proc Natl Acad Sci U S A 2020;117(21):11368-11378

71. Bush ML, Thompson R, Irungu C, Ayugi J. The role of telemedicine in auditory rehabilitation: a systematic review. Otol Neurotol 2016;37(10): 1466-1474

72. Meeuws M, Pascoal D, Janssens de Varebeke S, De Ceulaer G, Govaerts PJ. Cochlear implant telemedicine: remote fitting based on psychoacoustic self-tests and artificial intelligence. Cochlear Implants Int 2020;21(05):260-268

73. Kuzovkov V, Yanov Y, Levin S et al. Remote programming of MED-EL cochlear implants: users' and professionals' evaluation of the remote programming experience. Acta Otolaryngol 2014; 134(07):709-716

74. Luryi AL, Tower JI, Preston J, Burkland A, Trueheart CE, Hildrew DM. Cochlear implant mapping through telemedicine - a feasibility study. Otol Neurotol 2020;41(03):e330-e333
75. Slager HK, Jensen J, Kozlowski K et al. Remote programming of cochlear implants. Otol Neurotol 2019;40(03):e260-e266

76. Shayman CS, Ha YM, Raz Y, Hullar TE. Geographic disparities in US Veterans' access to cochlear implant care within the Veterans Health Administration System. JAMA Otolaryngol Head Neck Surg 2019;145(10):889-896

77. Garber S, Ridgely MS, Bradley M, Chin KW. Payment under public and private insurance and access to cochlear implants. Arch Otolaryngol Head Neck Surg 2002;128(10):1145-1152

78. Kim J, Jeon S, Kim D, Shin Y. A review of contemporary teleaudiology: literature review, technology, and considerations for practicing. J Audiol Otol 2021;25(01):1-7

79. Bhutta MF, Swanepoel W, Fagan J. ENT from afar: opportunities for remote patient assessment, clinical management, teaching and learning. Clin Otolaryngol 2021;46(04):689-691 